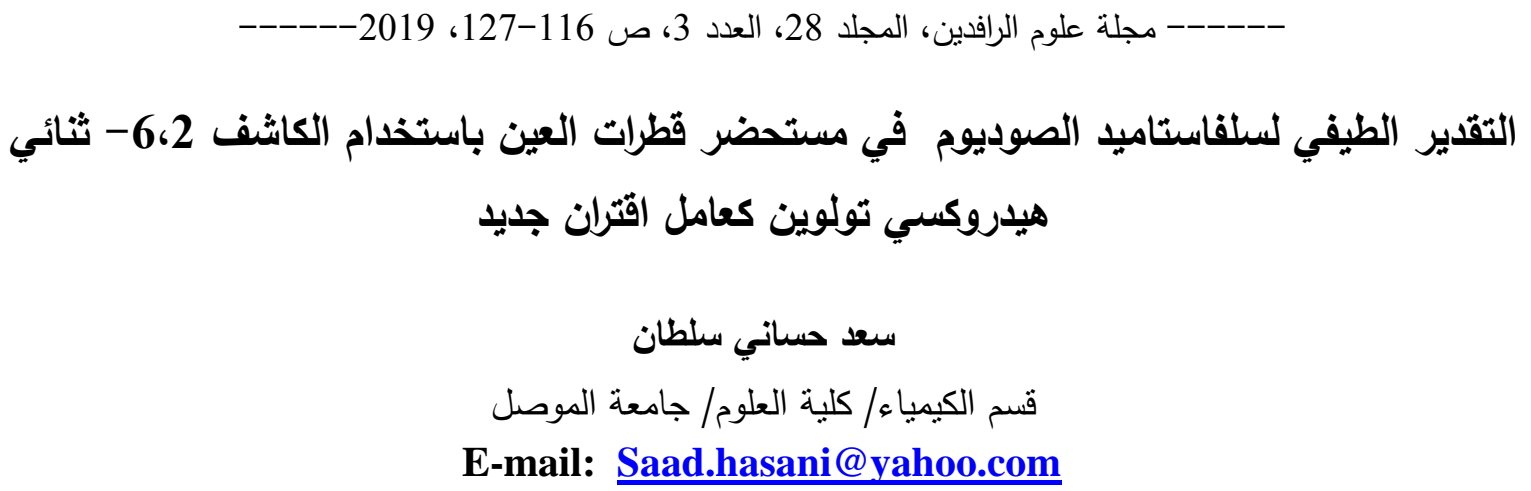

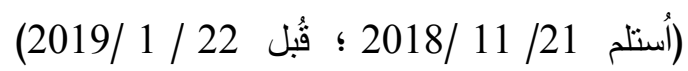

\begin{abstract}
الملخص
تضمن البحث وصف طريقة طيفية سهلة وحساسة لتقدير سلفاستاميد الصوديوم في المحلول المائي. اعتمدت الطريقة

المقترحة على ازوتة المركب الدوائي بوساطة نتريت الصوديوم في الوسط الحامضي لإعطاء ملح الديازونيوم المقابل للمركب الدوائي الذي يقترن مع الكاثف 6، 6- ثنائي هيدروكسي تولوين بوجود كاربونات الصوديوم لتكوين صبغة آزوية ملونة، ذائبة

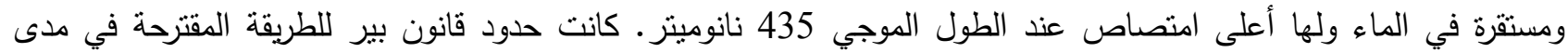

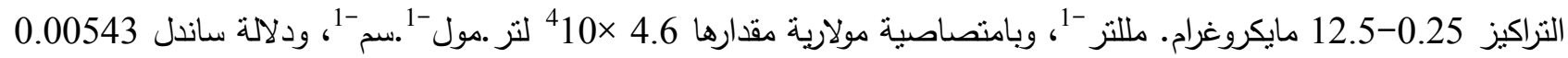

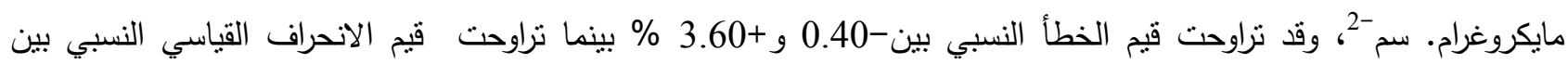

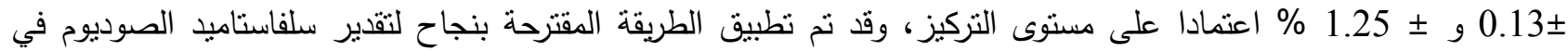
مستحضره الصيدلاني بشكل قطرة العين.
\end{abstract}

الكلمات الدالة: سلفاسيتاميد صوديوم، أزوتة واقتران، 6،2- ثنائي هيدروكسي تولوين، طريقة طيفية.

\title{
Spectrophotometric Assay of Sulphacetamide Sodium in Ophthalmic Preparation Using 2,6-Dihydroxytoluene as a New Coupling Agent
}

\author{
Saad H. Sultan \\ Department of Chemistry/ College of Science/ University of Mosul
}

\begin{abstract}
A simple and sensitive spectrophotometric method was described for the determination of sulphacetamide sodium (SAS) in aqueous solution. The proposed method depends on diazotization of drug compound using sodium nitrite in acidic medium to produce the corresponding diazonium salt which coupled with 2,6-dihydroxytoluene reagent in presence of sodium carbonate to form a colored water soluble and stable azo-dye, which has a maximum absorption at $435 \mathrm{~nm}$ with a molar absorptivity and Sandal's sensitivity of $4.6 \times 10^{4} 1 . \mathrm{mol}^{-1} \cdot \mathrm{cm}^{-1}$ and $0.00543 \mu \mathrm{g} \cdot \mathrm{cm}^{-2}$ respectively. Beer's law is valid over the concentration range $0.25-12.5 \mu \mathrm{g} \cdot \mathrm{ml}^{-1}$. The relative error is in between -0.40 and $+3.60 \%$ and the relative standard deviation from \pm 0.13 to $\pm 1.25 \%$ depending on the concentration level. The proposed method was applied successfully to determine sulphacetamide sodium in its pharmaceutical formulation as ophthalmic drops.
\end{abstract}

Keywords: Sulphacetamide sodium, diazotization and coupling, 2,6-dihydroxytoluene reagent, spectrophotometry. 


\section{المقدمة}

تعد أدوية السلفا من اقدم المضادات الحيوية المصنعة والمستخدمة في معالجة الالتهابات وتعرف ايضا بالسلفوناميدات،

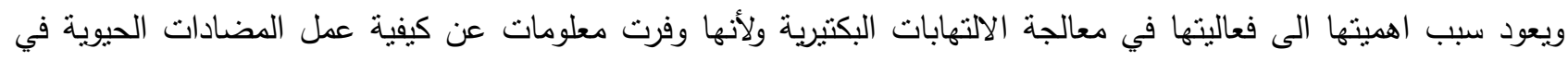
معالجة هذه الالتهابات (Csaky, 1979)، وعلى الرغم من اكتثاف مضادات حيوية اخرى مثل البنسلينات الا ان ادوية السلفا ماتزال نستخدم لحد الان في المجالات الطبية (Carey, 1996). ان اغلب ادوية السلفا تعمل كمضادات للأيض للبكتريا خلال تثتيطها للأنزيم البكتيري الذي يقوم بتحضير فيتامين حامض الفوليك من جزيئة بارا- امينو حامض البنزويك والذي يعد ضروريا

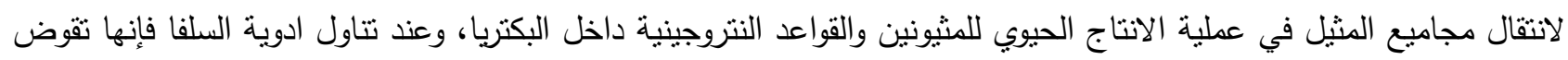

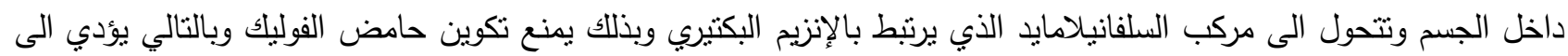

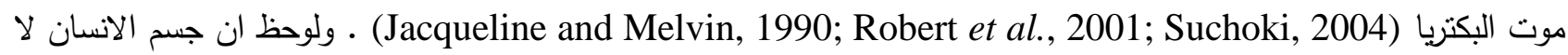
يتأثر بفعل ادوية السلفا وذلك لان الجسم لا يصنع حامض الفوليك بل يحصل عليه من الغذاء الذي ينتاوله وخاصة الخضراوات الورقية (Rang and Dale,1987).

ينتمي السلفاستاميد الى عائلة أدوية السلفا وعادة يوجد بشكل ملح الصوديوم ويدعى بـ (Soluble sulphacetamide)

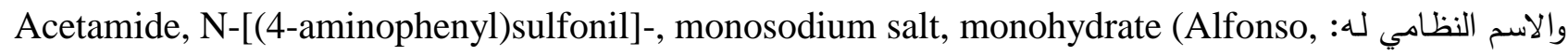
AK-Sulf, Albucid, Bleph-10, Cetamide, Sodium Sulamyd, Sulf-10, : 1995) Sulten-10 (Budavary, 2001)

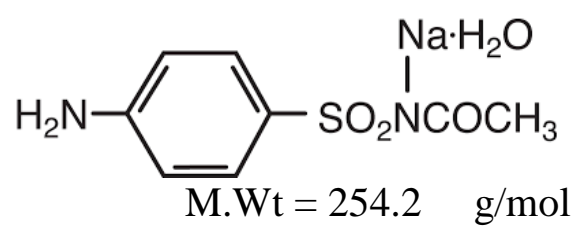

وهو عبارة عن مسحوق بلوري أبيض عديم الرائحة ينصهر عند درجة حرارة 257 مْ، ويذوب جدا في الماء وقليلا في

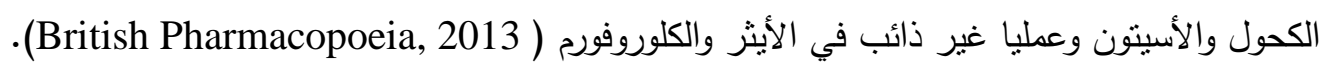
قدرّ سلفاستاميد الصوديوم باستخدام طرائق تحليلية مختلفة منها طرائق نسحيحية (Asahi et al., 1991) أو طرائق طيفية Othman and Kadder, 2006; AL-Nuri, and Al-Obaydi, 2009; Al-Uzari and Fadil, 2017; (Ahmed et al., 2017; Alsaffar, 2017; Chaluvaraju et al., 2010 (El-Raqehy et al., 2017) نظرا للأهمية الطبية لمركب السلفاسيتاميد الدوائي وبساطة الطرائق الطيفية في تقدير مختلف المركبات لذلك كان الهدف من البحث هو نطوير طريقة طيفية سهلة وسريعة لتقدير السلفاسيتاميد في المحلول المائي باستخدام تقاعلات الأزوتة والاقتران ذات الحساسية العالية وباستخدام كاشف اقتران جديدهو 2،6 كثائي هيدروكسي تولوين والذي لم يستخدم سابقا حسب معلوماتتا بوصفه كاثشا للأقتران، ثم تطبيق الطريقة المقترحة على مستحضره الصبدلاني بشكل قطرة العين واختبار مدى كفاءة الطريقة المقترحة 


\section{الجزء العملي}

الأجهزة المستخدمة: - (n)

تمت القياسات الطيفية باستخدام جهاز المطياف الضوئي ذي الشعاع المزدوج من نوع CECIL CE 7200 باستخدام خلايا من البلاستيك ذات مسار ضوئي مقداره 1 سم، وتم قياس الدالة الحامضية للمحاليل باستخدام جهاز قياس الدالة الحامضية من نوع TRANS BP3001، واستخدام ميزان حساس من نوع KERN لإجراء عمليات الوزن. المواد الكيمياوية المستخدمة: كانت جميع المواد الكيمياوية والكواثف التحليلية المستخدمة على درجة عالية من النقاوة. محلول سلفاسيتاميا الصوديوم SAS (50 مايكروغرام • مللتر -1):

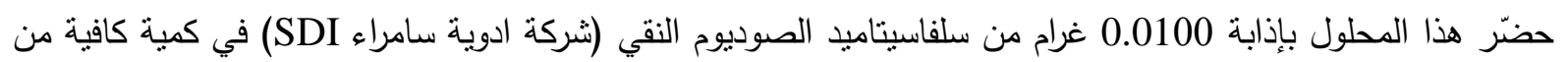
الماء المقطر ، ثم أُكمل الحجم إلى 200 مللتر بالماء المقطر باستخدام قنينة حجمية ونت حفظ المحلول في قنبينة معتمة. محلول حامض الهيدروكلوريك المخفف (1 عياري): يحضّر هذا المحلول بتخفيف 8.6 مللتر من الحامض المركّز (Thomas Baker) بالماء المقطر في قنتينة حجمية سعة 100 مللتر ويكمل الحجم إلى حد العلامة بالماء المقطر .

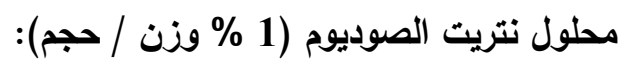
يحضّر هذا المحلول بإذابة غرام واحد من ملح نتريت الصوديوم (BDH) بكمية كافية من الماء المقطر إلى حد العلامة في قنينة حجمية سعة 100 مللتز

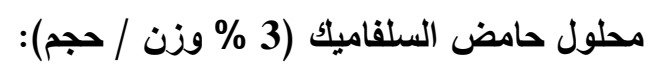
حُضِّر هذا المحلول بإذابة 3.0000 غرام من حامض السلفاميك (BDH) بالماء المقطر باستخدام قنينة حجمية سعة 100 مللتز

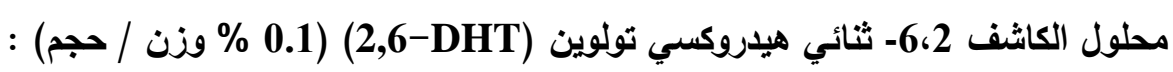
حضر هذا المحلول بإذابة 0.1000 غرام من الكاشف 6، 6- ثنائي هيدروكسي تولوين (Fluka) في كمية كافية من الماء

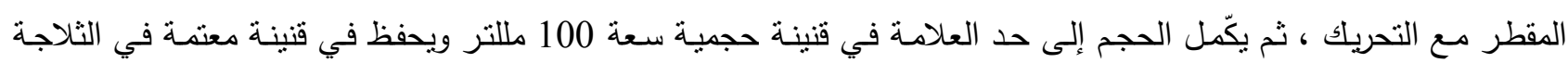
ويكون مستقرا لمدة عشرة أيام.

محلول كاريونات الصوديوم (1 مولاري): بحضّر هذا المحلول بإذابة 10.6000 غرام من كاربونات الصوديوم (Sigma) في 100 مللتر من الماء المقطّر باستخدام قنينة حجمية. محاليل مواد السواغ (10 مايكروغرام ـ مللتر -10): تحضّر هذه المحاليل بإذابة 0.0100 غرام من المواد المضافة في 100 مللتز من الماء المقطر باستخدام قنينة حجمية، ثم مأم يؤخذ 10 مللتر من هذا المحلول ويخفف بالماء المقطر باستخدام قنبنة حجمية سعة 100 مللتز . محلول المستحضر الدوائي لقطرة العين Apisulfa-20 ( 50 مايكروغرام . مللتر -1): حضرّ هذا المحلول وذلك بمزج محتويات ثلاث عبوات من قطرة العين (Apisulf-20) من إنتاج شركة عمّان للصناعات الدوائية/ الأردن (1 مللتر من محلول القطرة يحتوي على 200 مليغرام من سلفاسيتاميد الصوديوم) وأخذ 1 مللتز من المحلول

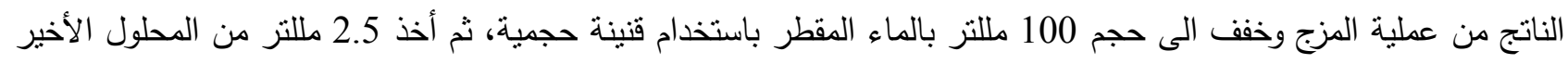
وخفف بالماء المقطر الى حجم 100 مللتر باستخدام قنينة حجمية. 


\section{النتائج والمناقشة}

تمت دراسة تأثير مختلف الظروف المؤثرة على امتصاص الصبغة المتكّونة وذلك باستخدام 1.0 مللتز من محلول

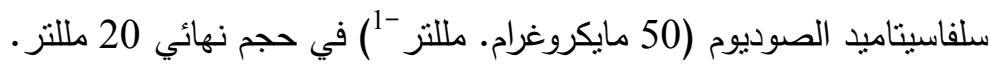

\section{المبدأ العام للطريقة}

تعتمد الطريقة المقترحة على أزوتة المركب الدوائي سلفاسيتاميد الصوديوم بواسطة نتريت الصوديوم في وسط حامضي لتكوين ملح الدايازونيوم الذي يقترن مع الكاشف 6، 6- ثنائي هيدروكسي تولوين ليعطي صبغة الآزو ذات اللون الأصفر وكما هو موضح في المعادلات الآتية:

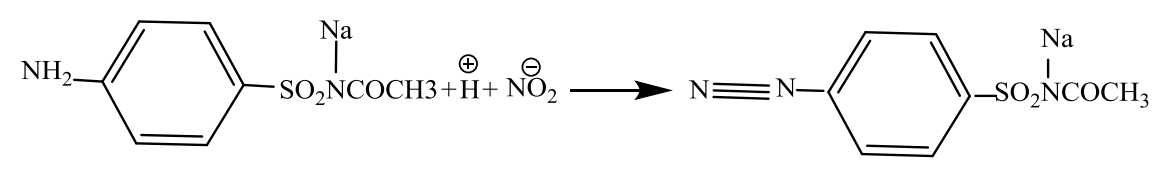

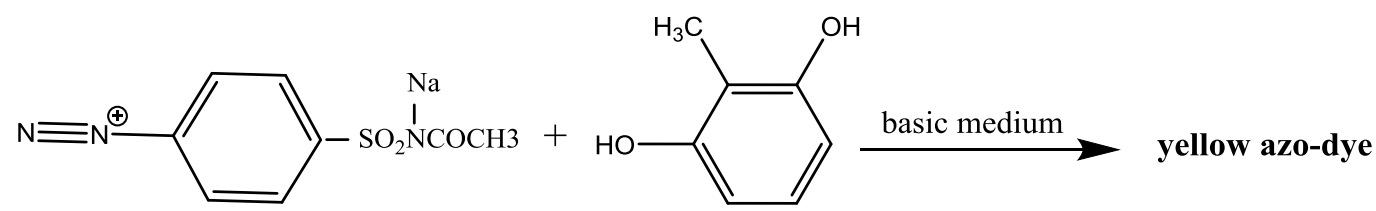

\section{تأثير نوعية وكمية الحامض المستخلم في عملية الأزوتة} إن من منطلبات عملية الأزوتة هو وجود حامض لتكوين ملح الدايازونيوم حيث تمت دراسة فئه تأثير إضافة نوعيات وكميات مختلفة من الحوامض، النتائج في (الجدول 1) تنيّن أن إضافة 1.0 مللتز من حامض الهيدروكلوريك بتركيز 1 عياري أعطى أفضل الفيل

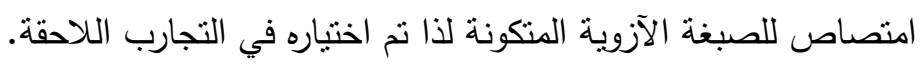

الجدول 1: تأثير نوعية وكمية الحامض في تفاعل الأزوتة

\begin{tabular}{|c|c|c|c|c|c|c|}
\hline \multirow{2}{*}{$\begin{array}{l}\text { Acid used, } \\
\text { (1N) soln. }\end{array}$} & \multicolumn{6}{|c|}{ Absorbance / mLof acid added } \\
\hline & 0.0 & 0.5 & 1.0 & 1.5 & 2.0 & 2.5 \\
\hline $\mathrm{HCl}$ & \multirow{4}{*}{0.388} & 0.417 & 0.428 & 0.425 & 0.422 & 0.418 \\
\hline $\mathrm{HNO}_{3}$ & & 0.419 & 0.425 & 0.420 & 0.410 & 0.391 \\
\hline $\mathrm{H}_{3} \mathrm{PO}_{4}$ & & 0.421 & 0.417 & 0.415 & 0.405 & 0407 \\
\hline $\mathrm{CH}_{3} \mathrm{COOH}$ & & 0.413 & 0.418 & 0.422 & 0.420 & 0.415 \\
\hline
\end{tabular}

تأثير كمية نتريت الصوديوم والزمن

تمت دراسة تأثنير كمية نتريت الصوديوم اللازمة في عملية أزوتة سلفاسيتاميد الصوديوم مع الزمن و (الجدول 2) يوضح أن أن

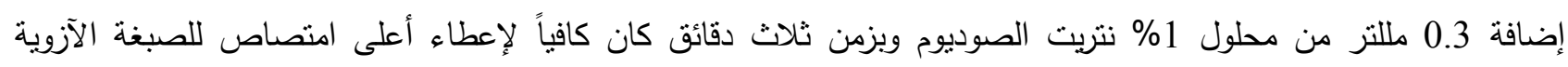
المتكونة لذلك فقد تم اعتماده في التجارب اللاحقة. 
الجدول 2: تأثير كمية نتريت الصوديوم والزمن في تفاعل الأزوتة

\begin{tabular}{|c|c|c|c|c|c|}
\hline \multirow{2}{*}{$\begin{array}{c}\text { mL of NaNO } \\
\text { (1\%) soln. }\end{array}$} & \multicolumn{5}{|c|}{ Absorbance / Standing time( min.) } \\
\cline { 2 - 6 } & $\mathbf{1}$ & $\mathbf{3}$ & $\mathbf{5}$ & $\mathbf{7}$ & $\mathbf{1 0}$ \\
\hline $\mathbf{0 . 1}$ & 0.420 & 0.423 & 0.424 & 0.441 & 0.443 \\
\hline $\mathbf{0 . 2}$ & 0.424 & 0.430 & 0.441 & 0.427 & 0.427 \\
\hline $\mathbf{0 . 3}$ & 0.438 & 0.439 & 0.437 & 0.430 & 0.431 \\
\hline $\mathbf{0 . 4}$ & 0.437 & 0.432 & 0.430 & 0.429 & 0.425 \\
\hline $\mathbf{0 . 5}$ & 0.421 & 0.430 & 0.435 & 0.421 & 0.416 \\
\hline
\end{tabular}

تأثير كمية حامض السلفاميك والزمن

إن كمية حامض النتروز المتبقية بعد عملية الأزوتة غير مرغوب بها إذ تعمل على نترزة عامل الاقتران مما يقلل من فعاليته

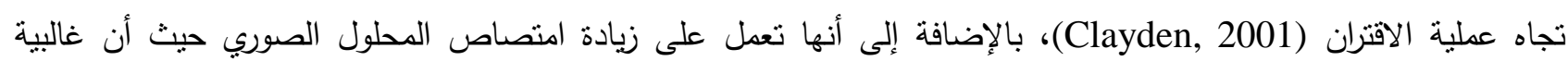
مركبات النايتروزو صفراء اللون (Baum and Scaife, 1980) ، لذلك تم استخدام حامض السلفاميك في إزالة المتبقي من حامض النتروز وذلك من خلا اختزاله إلى غاز النيتروجين الخامل(Brown et al., 1981) وكما هو موضح في المعادلة الآتية:

$$
\mathrm{HNO}_{2}+\mathrm{H}_{2} \mathrm{NSO}_{3} \mathrm{H} \longrightarrow \mathrm{H}_{2} \uparrow+\mathrm{H}_{2} \mathrm{SO}_{4}+\mathrm{H}_{2} \mathrm{O}
$$

النتائج المثبتة في (الجدول 3) نبين أن استخدام 0.3 مللتر من محلول 3 \% حامض السلفاميك مع زمن تحريك مقداره خمس دقائق يكون كافياً لإعطاء أعلى امتصاص للصبغة الازوية المتكونة مع قيمة امتصاص قليلة للمحلول الصوري لذانلك اعتماده في التجارب اللاحقة.

الجدول 3: تأثير حامض السلفاميك والزمن على الامتصاص

\begin{tabular}{|c|c|c|c|c|c|c|}
\hline \multirow{2}{*}{$\begin{array}{c}\text { mL of (3\%) } \\
\text { sulphamic } \\
\text { acid, soln. }\end{array}$} & \multirow{2}{*}{ Variable } & \multicolumn{5}{|c|}{ Absorbance / Standing time (min) } \\
\cline { 3 - 7 } & & $\mathbf{1}$ & $\mathbf{3}$ & $\mathbf{5}$ & $\mathbf{7}$ & $\mathbf{1 0}$ \\
\hline $\mathbf{0 . 1}$ & $\mathbf{S}$ & 0.416 & 0.464 & 0.438 & 0.322 & 0.300 \\
& $\mathbf{B}$ & 0.485 & 0.473 & 0.328 & 0.295 & 0.289 \\
\hline \multirow{2}{*}{$\mathbf{0 . 2}$} & $\mathbf{S}$ & 0.397 & 0.438 & 0.432 & 0.423 & 0.423 \\
& $\mathbf{B}$ & 0.027 & 0.028 & 0.023 & 0.022 & 0.024 \\
\hline \multirow{2}{*}{$\mathbf{0 . 3}$} & $\mathbf{S}$ & 0.429 & 0.442 & 0.451 & 0.449 & 0.445 \\
& $\mathbf{B}$ & 0.023 & 0.022 & 0.021 & 0.020 & 0.014 \\
\hline \multirow{2}{*}{$\mathbf{0 . 4}$} & $\mathbf{S}$ & 0.416 & 0.423 & 0.433 & 0.435 & 0.433 \\
& $\mathbf{B}$ & 0.028 & 0.017 & 0.019 & 0.017 & 0.016 \\
\hline \multirow{2}{*}{$\mathbf{0 . 5}$} & $\mathbf{S}$ & 0.418 & 0.437 & 0.430 & 0.429 & 0.428 \\
& $\mathbf{B}$ & 0.026 & 0.015 & 0.015 & 0.017 & 0.013 \\
\hline
\end{tabular}

$\mathrm{S}=$ Sample , B = Blank

تأثير كمية عامل الاقتران 6،2 - ثنائي هيدروكسي تولوين تمت دراسة تأثير كمية عامل الاقتران على امتصاص الصبغة الازوية المتكونة، (الجدول 4) يبين أن إضافة 0.5 مللتر من

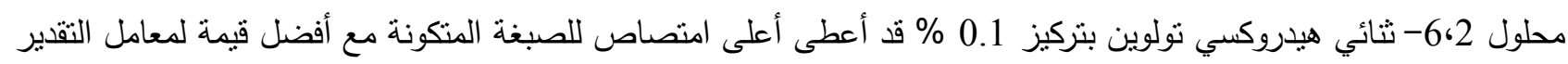
لذلك فقد نم اختياره للتجارب اللاحقة. 
الجدول 4: تأثير كمية عامل الاقتران على الامتصاص

\begin{tabular}{|c|c|c|c|c|c|c|c|c|c|}
\hline \multirow{2}{*}{$\begin{array}{l}\text { mL of } 2,6- \\
\text { DHT }(0.1 \%) \\
\text { soln. }\end{array}$} & \multicolumn{8}{|c|}{ Absorbance / $\mu \mathrm{g}$ of SAS } & \multirow{2}{*}{$\mathbf{R}^{2}$} \\
\hline & 25 & 50 & 75 & 100 & 125 & 150 & 175 & 200 & \\
\hline 0.1 & 0.116 & 0.312 & 0.516 & 0.729 & 0.885 & 1.118 & 1.470 & 1.580 & 0.9920 \\
\hline 0.25 & 0.241 & 0.445 & 0.643 & 0.860 & 1.050 & 1.266 & 1.496 & 1.612 & 0.9977 \\
\hline 0.5 & 0.242 & 0.442 & 0.668 & 0.864 & 1.078 & 1.270 & 1.502 & 1.628 & 0.9979 \\
\hline 1 & 0.248 & 0.451 & 0.663 & 0.868 & 1.084 & 1.274 & 1.45 & 1.598 & 0.9974 \\
\hline 1.5 & 0.256 & 0.462 & 0.665 & 0.895 & 1.095 & 1.282 & 1.482 & 1.596 & 0.9959 \\
\hline
\end{tabular}

تأثير نوعية وكمية القاعدة

بيّنت التجارب التمهيدية للتفاعل أن الصبغة الآزوية تتكون في الوسط القاعدي. لذلك تمت دراسة تأثنير نوعية وكمية محاليل قاعدية قوية وضعيفة مختلفة على امنصاص الصبغة المتكونة والنتائج في (الجدول 5) نبيّن أن استخدام 1.5 مللتز من كاربونات الصوديوم (pH=9.43) قد أعطى أفضل امتصاص للصبغة الآزوية المتكونة لذلك فقد نم اعنماده في التجارب اللاحقة. الجدول 5: تأثير نوعية وكمية القواعد على الامتصاص

\begin{tabular}{|l|c|c|c|c|c|c|c|}
\hline \multirow{2}{*}{ Base used (1M) } & \multicolumn{5}{|c|}{ Absorbance / $\mathbf{~ m L}$ of base added } & \multirow{2}{*}{$\begin{array}{c}\text { max } \\
\text { range }\end{array}$} & \multirow{2}{*}{ pH range } \\
\cline { 2 - 6 } & $\mathbf{1 . 0}$ & $\mathbf{1 . 5}$ & $\mathbf{2 . 0}$ & $\mathbf{2 . 5}$ & $\mathbf{3 . 0}$ & & \\
\hline $\mathbf{N a O H}$ & 0.191 & 0.452 & 0.432 & 0.363 & 0.323 & $431-438$ & $2.31-12.71$ \\
\hline $\mathbf{K O H}$ & 0.199 & 0.436 & 0.420 & 0.351 & 0.315 & $435-437$ & $2.37-12.89$ \\
\hline $\mathbf{N a}_{\mathbf{2}} \mathbf{C O}_{\mathbf{3}}$ & 0.472 & 0.499 & 0.498 & 0.495 & 0.490 & $434-436$ & $7.06-10.17$ \\
\hline $\mathbf{N a H C O}_{\mathbf{3}}$ & 0.271 & 0.453 & 0.485 & 0.495 & 0.489 & $407-436$ & $5.27-9.18$ \\
\hline
\end{tabular}

تأثير إضافة المواد الفعالة سطحياً تمت دراسة نأثير إضافة 3 ملتزر من ثلاثة أنواع مختلفة من المواد الفعالة سطحياً (المتعادلة مثل Triton X-100 وسالبة منل SDS وموجبة مثل CPC إلى محيط التفاعل وبتسلسلات مختلفة على امتصاص الصبغة المتكونة، وقد دلت النتائج المستحصلة أن استخدام عوامل الثد السطحي أدى إلى تقليل الامتصاصية لذلك فقد نم استبعادها في التجارب اللاحقة.

زمن استقراريه الصبغة المتكونة تمت دراسة استقراريه الصبغة المتكونة عند درجة حرارة المختبر ( 27 ـ 2ْم) وذلك بقياس الامتصاص مقابل المحلول

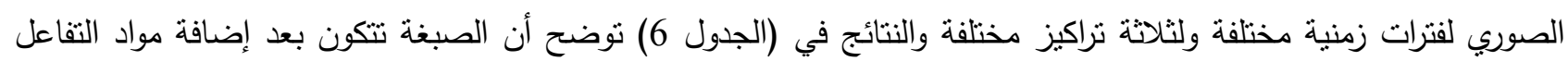
مباشرةً وتبقى مستقرة لمدة ساعتين على الأقل. 
الجدول 6: زمن استقرارية الصبغة المتكونة

\begin{tabular}{|c|c|c|c|}
\hline \multirow{2}{*}{ Time (min .) } & \multicolumn{3}{|c|}{ Absorbance/ $\boldsymbol{\mu g}$ of SAS } \\
\cline { 2 - 4 } & $\mathbf{2 5}$ & $\mathbf{5 0}$ & $\mathbf{2 0 0}$ \\
\hline After dilution & 0.265 & 0.499 & 1.886 \\
\hline $\mathbf{5}$ & 0.265 & 0.503 & 1.885 \\
\hline $\mathbf{1 0}$ & 0.265 & 0.503 & 1.885 \\
\hline $\mathbf{1 5}$ & 0.266 & 0.503 & 1.884 \\
\hline $\mathbf{2 0}$ & 0.266 & 0.503 & 1.883 \\
\hline $\mathbf{2 5}$ & 0.267 & 0.502 & 1.883 \\
\hline $\mathbf{3 0}$ & 0.268 & 0.503 & 1.880 \\
\hline $\mathbf{3 5}$ & 0.269 & 0.503 & 1.878 \\
\hline $\mathbf{4 0}$ & 0.270 & 0.503 & 1.877 \\
\hline $\mathbf{4 5}$ & 0.271 & 0.503 & 1.875 \\
\hline $\mathbf{5 0}$ & 0.272 & 0.502 & 1.875 \\
\hline $\mathbf{5 5}$ & 0.270 & 0.502 & 1.874 \\
\hline $\mathbf{6 0} \mathbf{( 1 h r}$ ) & 0.269 & 0.502 & 1.873 \\
\hline $\mathbf{2 ~ h r}$. & 0.267 & 0.501 & 1.860 \\
\hline
\end{tabular}

طيف الامتصاص النهائي

عند مفاعلة المركب الدوائي سلفاسيتاميد الصوديوم مع نتريت الصوديوم في الوسط الحامضي يتكون ملح الدايازونيوم الذي يقترن مع الكاثف 2، 6- ثنائي هيدروكسي تولوين في الوسط القاعدي لتكوين صبغة صفراء مستقرة وذائبة في الماء تعطي أعلى لنى

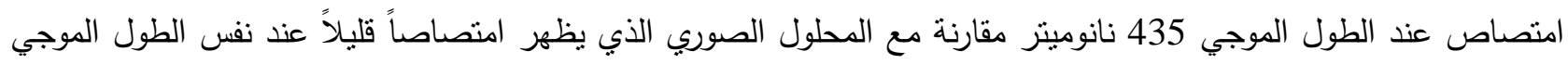
والثكل (1) يييّن طيف الامتصاص النهائي للصبغة المنكونة مقابل المحلول الصوري وطيف امتصاص الصبغة مقابل الماء. المقطر وطيف امتصاص المحلول الصوري مقابل الماء المقطر .

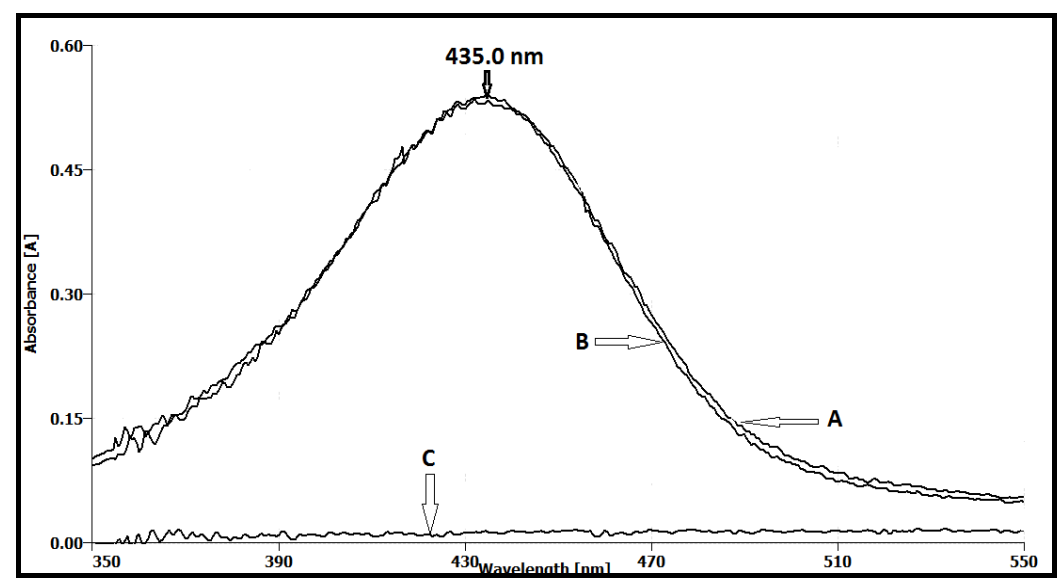

الثكل 1: طيف الامتصاص لـ 50 مايكروغرام / 20 مللتر من سلفاسيتاميد الصوديوم مقاساً

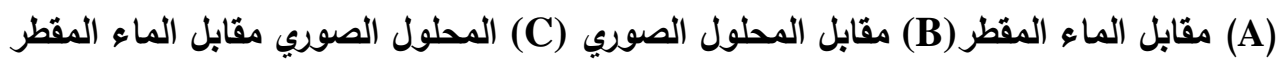

طريقة العمل المعتمدة والمنحنى القياسي ينت تحضير المنحنى القياسي لطريقة العمل بعد تثثيت الظروف المثلى عملياً لتقدير سلفاسيتاميد الصوديوم وكما يلي: 
تضاف حجوم متزايدة من محلول سلفاسيتاميد الصوديوم بحيث تحتوي على (2-300) مايكروغرام إلى سلسلة من القناني الحجمية

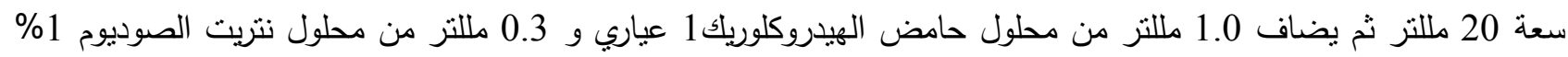

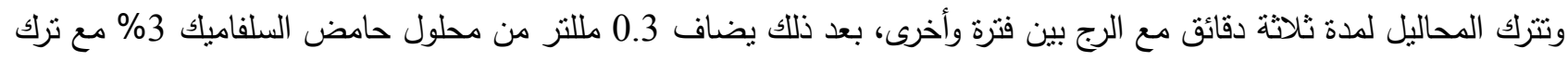
المحاليل لمدة خمس دقائق مع التحريك للتخلص من الفائض من نتريت الصوديوم، بعد ذللك يضاف 0.5 مللتر من محلول

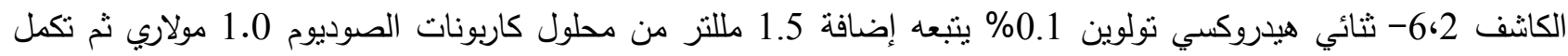
الحجوم بالماء المقطر إلى حد العلامة ويقاس الامتصاص للمحاليل مقابل المحلول الصوري عند الطول الموجي 435 نانوميتر، والمنحنى القياسي الموضح في الثكل (2) يكون موافقاً لقانون بير في مدى التراكيز 5 - 250 مايكروغرام/20 مللتر (0.25-

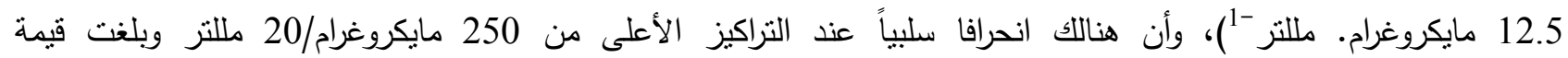

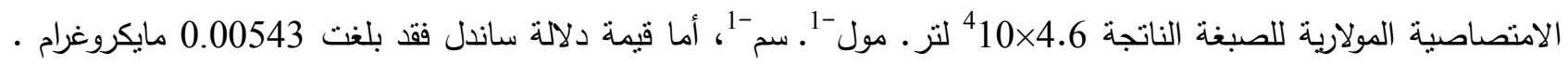

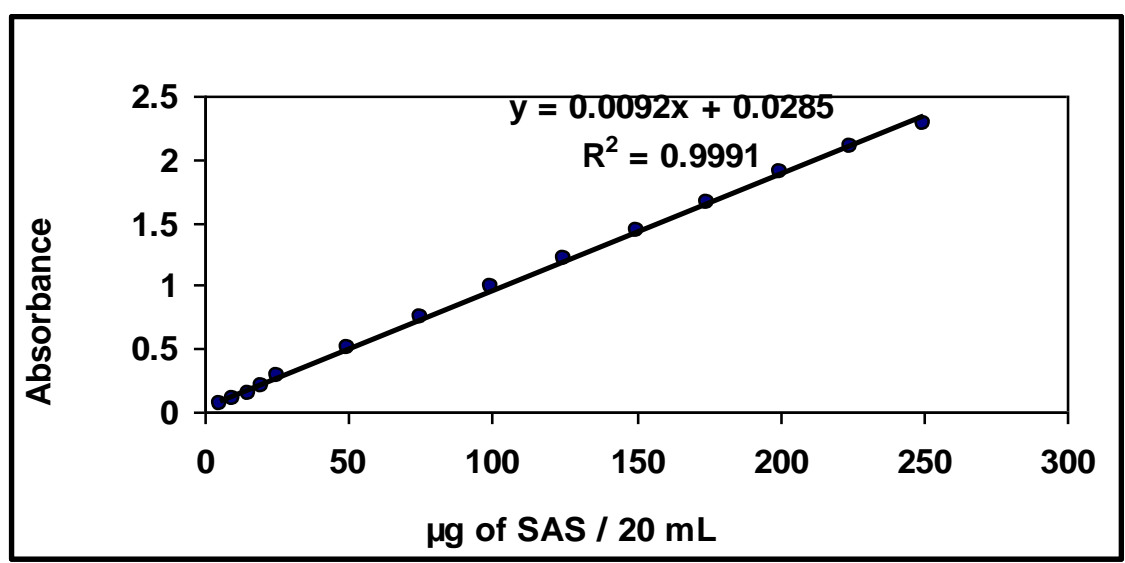

الشكل 2: المنحنى القياسي لتقدير سلفاسيتاميد الصوديوم المعامل وفق الطريقة المقترحة

وقد تم حساب قيمة حد الكثف (LOD) وقيمة حد التقدير الكمي (LOQ) (Valcarcel, 2000) للطريقة المقترحة وذلك بقياس الامتصاص لعشرة محاليل صورية مقابل الماء المقطر عند الطول الموجي 435 نانوميتر وحسب طريقة العمل المنلى، حيث بلغت قيمة حد الكثف 0.03627 مايكروغرام/ مللتر وقيمة حد التقدير الكمي 0.12093 مايكروغرام/مللتر . طبيعة الصبغة المتكوّنة لغرض معرفة نسبة ارتباط ملح الدايازونيوم المقابل لسلفاسيتاميد الصوديوم المختزل مع كاثف 6، 6- ثنائي هيدروكسي تولوين فقد تم تطبيق طريقة التغييرات المستمرة (طريقة جوب) وكما في الثكل (3). 


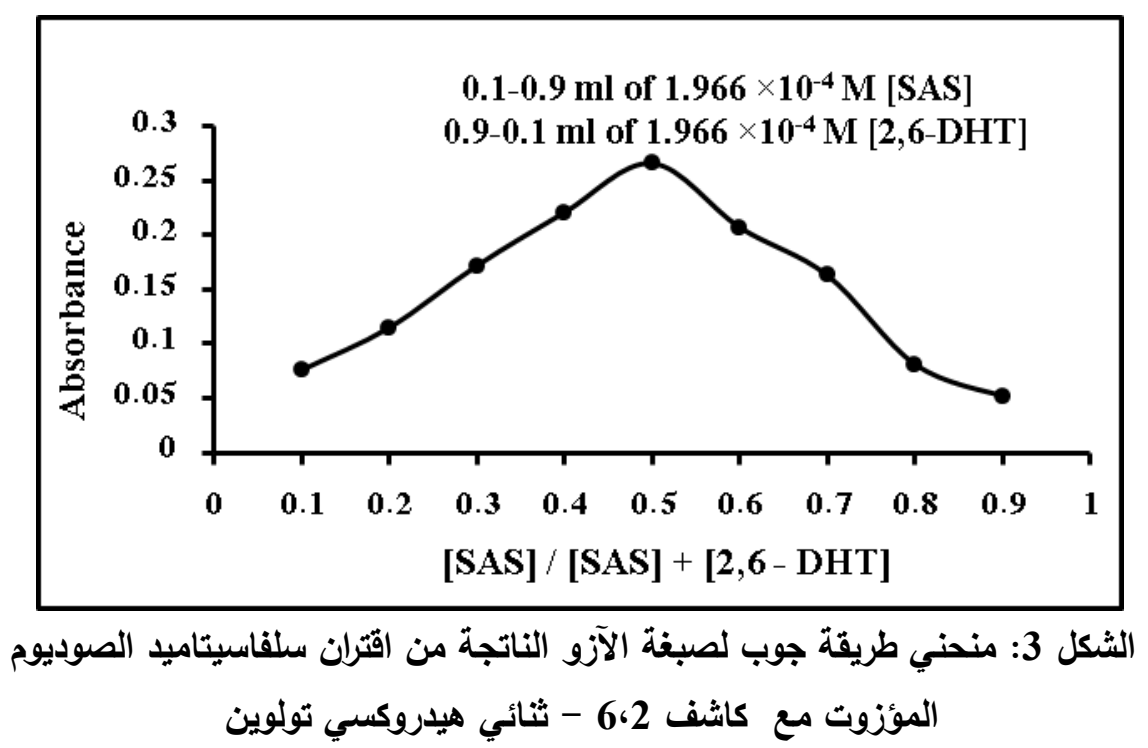

إن الثكل (3) يوضح ان نسبة ارتباط سلفاسيتاميد الصوديوم المؤزوت مع الكاشف 6،2 - ثنائي هيدروكسي نولوين

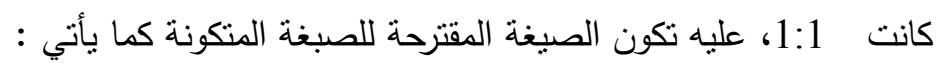<smiles>CC(=O)NNNc1ccc(S(=O)(=O)O[Na])cc1</smiles>

Yellow azo dye $\left(\lambda_{\max }=435 \mathrm{~nm}\right)$

تأثير المتداخلات

تمت دراسة تأثير بعض المركبات والتي يمكن أن تضاف عند تصنيع المستحضر الدوائي لسلفاسيتاميد الصوديوم بشكل قطرة

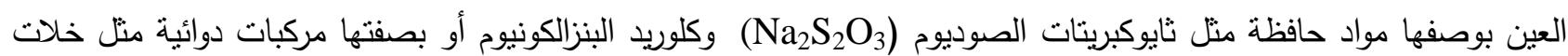

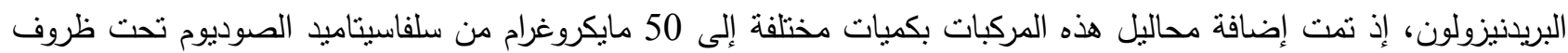

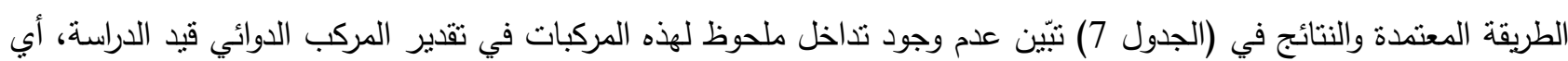
إمكانية تقدير سلفاسيتاميد الصوديوم في مستحضراته الدوائية بوجود هذه المركبات باستخدام الطريقة المقترحة.

الجدول 7: تأثير المضافات على تقدير سلفاسيتأميد الصوديوم حسب الطريقة المقترحة

\begin{tabular}{|c|c|c|c|c|}
\hline \multirow{2}{*}{ Additives } & \multicolumn{4}{|c|}{$\begin{array}{c}\text { Recovery (\%) of } 50 \mu \mathrm{g} \text { SAS / } \mu \mathrm{g} \text { of foreign compound } \\
\text { added }\end{array}$} \\
\hline & 2.5 & 5.0 & 7.5 & 10.0 \\
\hline $\begin{array}{c}\text { Benzalconium } \\
\text { chloride }\end{array}$ & 99.0 & 99.2 & 98.8 & 99.2 \\
\hline $\begin{array}{c}\text { Sodium } \\
\text { thiosulfate }\end{array}$ & 98.8 & 98.6 & 97.6 & 99.6 \\
\hline $\begin{array}{c}\text { Prednisolone } \\
\text { acetate }\end{array}$ & 96.6 & 97.4 & 96.6 & 97.0 \\
\hline
\end{tabular}


الجزء التطبيقي

تم تطبيق الطريقة المقترحة في تقدير سلفاسيتاميد الصوديوم في المستحضر الصيدلاني المتوفر في الأسواق المحلية على شكل قطرة عين، وذلك بأخذ ثلاثة تراكيز مختلفة (25 و 50 و 200 ) مايكروغرام من سلفاسيتاميد الصوديوم ثم تطبق خطوات

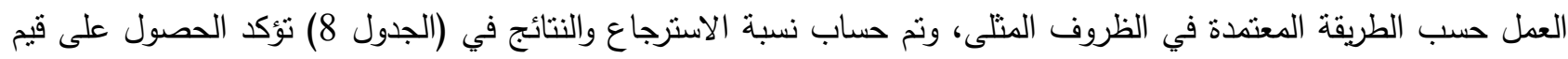
استرجاعية جيدة للسلفاسيتاميد الصوديوم في المستحضر الصيدلاني قطرة العين نوع Apisulfa-20. الجدول 8: إيجاد النسبة المئوية للاسترجاع لتقدير سلفاسيتاميد الصوديوم في قطرة العين

\begin{tabular}{|c|c|c|c|}
\hline $\begin{array}{c}\text { Pharmaceutical } \\
\text { preparation }\end{array}$ & $\begin{array}{c}\text { Amount } \\
\text { taken, } \boldsymbol{\mu g}\end{array}$ & $\begin{array}{c}\text { Amount } \\
\text { measured, } \boldsymbol{\mu g}\end{array}$ & Recovery*, \% \\
\hline Apisulfa-20 Steril Eye Drops (20\%) & 25 & 25.1 & 100.4 \\
\cline { 2 - 4 } Amman pharmaceutical Industries & 50 & 49.1 & 98.2 \\
\cline { 2 - 4 } Co. Ltd (Jordan) & 200 & 193.6 & 96.8 \\
\hline
\end{tabular}

*Average of five determinations

كفاعة الطريقة المقترحة لغرض إثبات كفاءة الطريقة المقترحة ونجاحها في تقدير سلفاسيتاميد الصوديوم وخلوها من تداخلات المواد المضافة في المستحضر الصيدلاني فقد طبقت طريقة الإضافة القياسية على تركيزين مختلفين من المستحضر الصيدلاني وذللك لعدم نوفر مستلزمات الطريقة القياسية المعتمدة لتقدير السلفاسيتاميد (والتي تتضمن التسحيح المجهادي للسلفاسيتاميد مع محلول نتريت الصوديوم) وتشير النتائج المبينة في الثكل (4) و (الجدول 9) أن طريقة الإضافة القياسية متفقة بشكل جيد مع الطريقة المقترحة مما بدل على أن الطريقة ذات انتقائية جيدة.

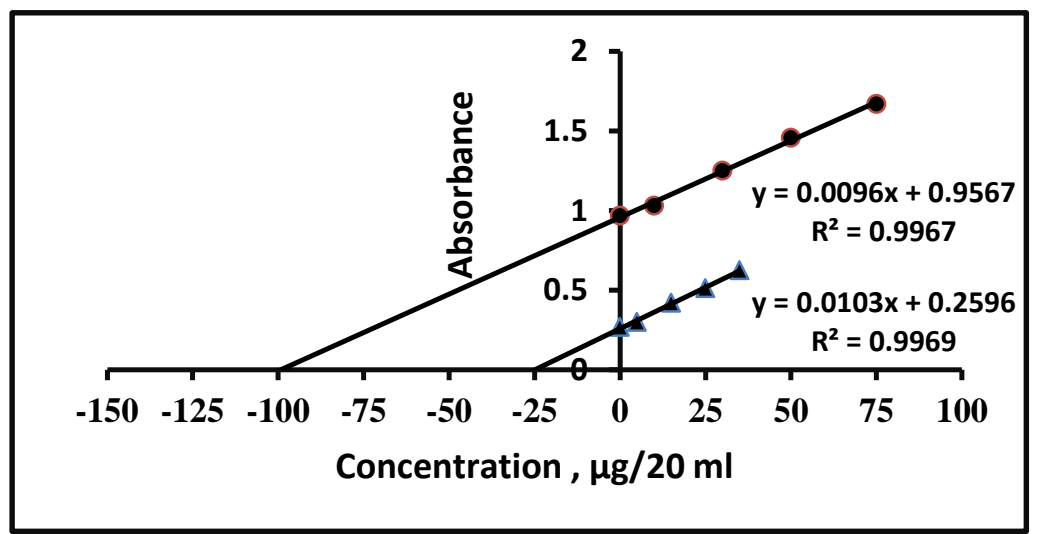

الثكل 4: منحنيات الاضافة القياسية لتقدير سلقاسيتاميد الصوديوم في المستحضر الصيدلاني

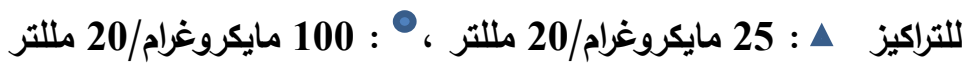

الجدول 9: نتائج استرجاعية سلفاسيتاميد الصوديوم في المستحضر الصيدلاني بالطريقة المقترحة والاضافة القياسية

\begin{tabular}{|c|c|c|c|}
\hline $\begin{array}{c}\text { Pharmaceutical } \\
\text { preparation }\end{array}$ & \multirow{2}{*}{$\begin{array}{c}\text { Amount } \\
\text { taken, } \boldsymbol{\mu g}\end{array}$} & \multicolumn{2}{|c|}{ Recovery*, \% } \\
\cline { 3 - 4 } \cline { 4 - 4 } & & Present method & $\begin{array}{c}\text { Standard } \\
\text { addition method }\end{array}$ \\
\cline { 3 - 4 } $\begin{array}{c}\text { Apisulfa-20 Steril Eye Drops } \\
\text { (20\%) Amman pharmaceutical } \\
\text { Industries Co. Ltd (Jordan) }\end{array}$ & 25 & 100.4 & 100.8 \\
\cline { 3 - 4 } & 100 & 99.8 & 99.6 \\
\hline
\end{tabular}


مقارنة الطريقة الحالية

لغرض معرفة مميزات الطريقة الحالية فقد تمت مقارنة قيم بعض المتغيرات التحليلية لها مع متغيرات طريقة طيفية أخرى وكما هو مبين في (الجدول 10).

الجدول 10: مقارنة بعض المتغيرات التحليلية للطريقة المقترحة مع طريقة طيفية أخرى

\begin{tabular}{|c|c|c|}
\hline Analytical parameter & Present method & Literature method* \\
\hline Reagent & 2,6- DHT & -Dihydroxybenzophenone 6,2 \\
\hline $\mathrm{pH}$ & 9.43 & basic \\
\hline Temperature $\left(\mathrm{C}^{\circ}\right)$ & R.T & R.T \\
\hline Development time (min.) & After dilution & After dilution \\
\hline$\lambda_{\max }(\mathrm{nm})$ & 435 & 497.5 \\
\hline 's law range $\left(\mu \mathrm{g} \cdot \mathrm{ml}^{-1}\right)$ Beer & $0.25-12.5$ & $25-1$ \\
\hline $\begin{array}{l}\text { Molar absorptivity } \\
\left(1 . \text { mole }^{-1} \cdot \mathrm{cm}^{-1}\right)\end{array}$ & $4.6 \times 10^{4}$ & $2.2 \times 10^{4}$ \\
\hline Stability of the color (hr.) & 2 & 1 \\
\hline Color of the dye & Yellow & Orange \\
\hline Nature of the dye & $1: 1$ & $1: 1$ \\
\hline Application of the method & Eye drop & Eye drop \\
\hline
\end{tabular}

*Al-Safar, 2017

النتائج في (الجدول 10) نبيّن أن الطريقة المقترحة الحالية تمنلك حساسية أعلى مقارنة مع الطريقة المنشورة، كما أن الصبغة المتكونة ذات استقرارية اكبر (ساعتين على الأقل) مما يتيح وقت كافي لإجراء عدة قياسات. الاستنتاجات

تم اقتراح طريقة طيفية سهلة وسريعة ودقيقة لتقدير سلفاسيتاميد الصوديوم في المحلول المائي بالاعتماد على أزوتة المركب

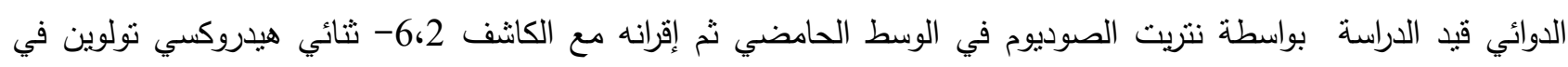
الوسط القاعدي لتكوين صبغة صفراء ذائبة في الماء ومستقرة وتعطي أعلى امتصاص عند الطول الموجي 435 نانوميتر • وتم

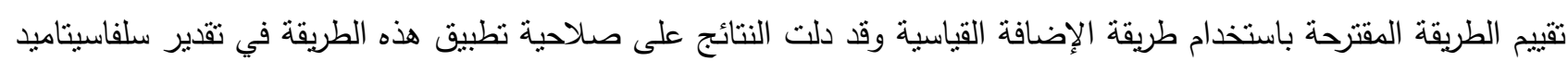
الصوديوم في المستحضر الدوائي قطرة العين (Apisulfa-20).

\section{المصادر}

Ahmed, S.; Anwar, N.; Sheraz, M.A.; Ahmad, I. (2017). Validation of a stability-indicating spectrophotometric method for the determination of sulphacetamide sodium in pure form and ophthalmic preparations. J. Pharm. Bioallied. Sci., 9(2),126-134.

Alfonso, R.G. (1995). "Remington: The Science and Practice of Pharmacy". 19 ${ }^{\text {th }}$ ed., Mack Publishing Co., Easton, pp.1272.

Al-Nuri, I.J.; Al-Obaydi, I.A. (2009). Direct determination of sulfacetamide sodium by derivative uv spectrophotometry. J. Raf. Sci., 20(4), 17-26.

Al-Sabha, T.N.; Hasan, M.A.; Ibrahim, H.A. (2014). Spectrophotometric assay of some nitrogen containing drugs in pharmaceutical formulation using p-chloranilic acid reagent. J. Advan. Chem. 9(1), 1798-1809.

AlSaffar, R.S. (2017). Development of spectrophotometric methods for determination of amoxicillin and sulphacetamide sodium -applications to pharmaceutical preparations. $\mathrm{Ph} . \mathrm{D}$. Thesis of Science, University of Mosul, Mosul, Iraq, pp.1-153. 
Al-Uzari, W.A.; Fadil, G. (2017). Spectrophotometric determination of sulfacetamide sodium in pharmaceutical preparation using 8-hydroxy-7-iodoquinoline-5-sulfonic acid as chromogenic reagent. Asian J. Chem., 29(4), 782-786.

Asahi, Y; Tanaka, M.; Sugimoto, M. (1991). Titration of sulfa drugs with perchloric acid in non aqueous solution. Bunseki Kagaku, 40(12), 889-893.

Baum, S. ; Scaife, C. (1980). "Chemistry a Life Science Approach". $2^{\text {nd }}$ ed., Macmillan Publishing Co. Inc., New York. 477 p.

British Pharmacopoeia, (2013). On CD-ROM, "System Simulation Ltd". The Stationary Office, London.

Brown, R.M.; Fry, R.; Moyers, J.L.; Nothway, S.J.; Denton, N.B.; Welson, G.S. (1981). Interference by volatile nitrogen oxides and transition-metal catalysis in the pre-concentration of arsenic and selenium as hydrides. Anal. Chem., 53, 1560-1566.

Budavari, S. (2001). "The Merck Index". 13 ${ }^{\text {th }}$ ed., Merk and Co., NJ, 8986 p.

Carey, F.A. (1996) ." Organic Chemistry". $5^{\text {th }}$ ed. The McGraw-Hill Company, New York. pp. 935936.

Chaluvaraju, K.C.; Bhat, K.I.; Zaranappa, (2010). Quantitative spectrophotometric determination of sulphacetamid sodium in bulk and in pharmaceutical dosage form. J. Pharm. Res., 3(1), 47-48.

Clayden, J.; Greeves, N.; Warren, S.; Wathers, P. (2001). "Organic Chemistry". $2^{\text {nd }}$ ed. Oxford University Press, London. 477 p.

Csaky, T.Z. (1979). "Cutting's Handbook of Pharmacology, The Actions and Uses of Drugs". $6^{\text {th }}$ ed., Appleton-Century-Crifts, New York. 1 p.

El-Raqehy, N.A.; Hegazy, M.A.; Abd El Hamid, G.; Tawfik, S.A. (2017). Validated chromatographic methods for the simultaneous determination of sulphacetamide sodium and prednisolone acetate in their ophthalmic suspension. J. Chromatogr. Sci. 55(10), 1000-1005.

Jacqueline, I.K.; Melvin, W. (1990). "Chemistry: General, Oranig, Biological". $2^{\text {nd }}$ ed., The McGraw-Hill, Inc., New York. 690 p.

Othman, N.S.; Kadder, R.M. (2006). Application of trifluoperazine hydrochloride as a chromogenic reagent for spectrophotometric determination of sulphacetamide sodium-application to ophthalmic preparations. Raf. J. Sci.,17(4), 92-103.

Rang, H.P.; Dale, M.M. (1987). "Pharmacology". Longman Group Ltd., London, pp.629-630.

Robert, L.C.; Katherine, J.D.; Joseph, J.T. (2001). "Principles and Applications of Inorganic, Organic and Biological Chemistry". $2^{\text {nd }}$ ed., WCB/McGraw- Hill Company, New York. $480 \mathrm{p}$.

Suchoki, J. (2004). "Conceptual Chemistry". Benjamin Cummings, New York. pp. 443-444.

Valcarcel, M. (2000). "Principles of Analytical Chemistry". Springer Verlag, Berlin, pp. 67-68.

Yadav, S.K.; Choubey, P.K.; Aqrawal, B.A.; Goyal, R.N. (2014). Carbon nanotube embedded poly 1,5-diaminonaphthalene modified pyrolytic graphite sensor for the determination of sulfacetamide in pharmaceutical formulations. Talanta., 118, 96-103. 